\title{
Measurement invariance across mother/child and father/child attachment relationships
}

\author{
Marília Fernandesa, Manuela Veríssimo (iD ${ }^{a}$, António J. Santos (iD) ${ }^{a}$ Olívia Ribeiroa, \\ Brian E. Vaughn (iD ${ }^{b}$, Marissa Gastelle $\mathbb{D D}^{c}$ and Kathryn A. Kerns $\mathbb{D}^{c}$ \\ aWilliam James Center for Research, ISPA- Instituto Universitário, Lisbon, Portugal; ${ }^{b}$ Family \& Child \\ Development, Auburn University, Auburn, AL, USA; 'Department of Psychological Sciences, Kent State \\ University, Kent, $\mathrm{OH}$, USA
}

\begin{abstract}
We examine the factorial structure of the Security Scale Questionnaire (SSQ), exploring measurement invariance across mother-father-child attachment relationships, child sex, and country. We used the new 21-item SSQ version that integrates both safe haven and secure base behaviors in a two factors structure. Participants were 457 children ( 224 girls and 233 boys), ranging from 9 to 14 years old $(M=10.84, S D=1.02)$ from Portuguese and USA samples. We confirmed the SSQ's two-factor structure, although four items were unrelated to the latent structure and excluded from the final model. Results showed that SSQ can be used to study both mother/child and father/child attachment relationships. Multi-group analyses suggested measurement invariance between boys and girls and between Portuguese and USA samples. Our findings suggest that the SSQ can be considered a valid and cost-effective tool to measure perceived attachment security in middle childhood for both mother/child and father/child relationships.
\end{abstract}

\section{ARTICLE HISTORY}

Received 19 January 2019

Accepted 26 December 2019

\section{KEYWORDS}

Attachment; middle childhood; cultural context; mother; father

Bowlby, Ainsworth and others (e.g. Ainsworth, 1989; Ainsworth, Blehar, Waters, \& Wall, 1978; Bowlby, 1982; Waters \& Cummings, 2000) defined attachment security in terms of the secure base phenomenon, that is, a pattern of interactions between an infant/child and a caregiver (usually a parent) indicating that the child uses him or her as a secure base from which to explore the local and more distant worlds (Secure Base) and seeks out the caregiver when the child is distressed, tired, or desirous of increased stimulation (Safe Haven). During infancy and toddlerhood, attachment relationships are based on the activation of a behavioral system that operates to help the child gain and maintain physical proximity and support from a caregiver (Bowlby, 1982). Most studies of early attachments evaluate individual differences in attachment quality using observational measures of parent-child interaction (e.g. the Strange Situation (SSP)-Ainsworth et al. 1978; or the Attachment Q-sort (AQS) - Waters \& Deane, 1985). Moreover, parents tend to be the primary attachment figures for their children throughout childhood and into 
adolescence (e.g. Levitt, Guacci-Franco, \& Levitt, 1993; Reid, Landesman, Treder, \& Jaccard, 1989; Seibert \& Kerns, 2009).

However, by middle childhood, the goals of secure base and safe haven support shift to reflect the child's growing physical, cognitive, and emotional competencies (see Fraley, 2002; Saarni, 1999; Van IJzendoorn \& Bakermans-Kranenburg, 2010; Waters, Hamilton, \& Weinfield, 2000). By middle childhood, parents and child are able to co-construct a cooperative partnership where both take responsibility for communicating and coordinating the behavioral components of a secure base relationship (e.g. Bowlby, 1982; Kerns, Brumariu, \& Seibert, 2011; Waters, Kondo-lkemura, Posada, \& Richters, 1991). At this point in development, the child's main goal is to ensure parents' readiness and availability to act in the child's best interests, rather than proximity per se (e.g. Kerns, Schlegelmilch, Morgan, \& Abraham, 2005).

Most attachment studies with middle childhood or early adolescent samples rely on data obtained directly from participants, using narrative measures (e.g. Attachment Story Completion Task; Bretherton, Ridgeway, \& Cassidy, 1990; Adult/Adolescent Attachment Interview, AAl; George, Kaplan, \& Main, 1996) or questionnaires (e.g. Security Scale; Kerns, Aspelmeier, Gentzler, \& Grabill, 2001) designed to elicit mental representations of attachment(s) to primary caregivers. These assessments presume that social cognitive growth allows children and adolescents to reflect more deeply on their relationship experiences and that their more advanced vocabulary allows them to articulate and communicate those experiences more precisely. The children included in this study were between the early stages of middle childhood and early adolescence; the range of ages suggested by Bowlby, 1982 as the time during which mental representations of attachment become consolidated and stable.

The Security Scale (SS; Kerns et al., 2001; Kerns, Klepac, \& Cole, 1996) is a widely used self-report questionnaire specifically designed for children between 8 and 14 years old. The SS assesses children's perceptions of specific attachment relationships (e.g. with each parent). The original scale contained 15 items and was intended to assess attachment security towards the mother or father. The central focus was on the degree to which the attachment figure served as a safe haven when needed. Thus, items assessed whether a child saw the caregiver as responsive, available, and reliable during times of stress (Kerns et al., 1996). Studies testing the reliability of the SS in different cultures and age groups in both clinical and community settings concluded that the scale demonstrated adequate internal consistency for both mothers and fathers, with Cronbach's alpha ranging from .64 to .93 (e.g. Al-Yagon, 2011; Granot \& Mayseless, 2001; Guttmann-Steinmettz, Shoshani, Farhan, Aliman, \& Hirschberger, 2012; Lieberman, Doyle, \& Markiewicz, 1999). In a recent meta-analysis, Brumariu, Madigan, Giuseppone, Abtahi, and Kerns (2018), reported that the SS was moderately stable over time, suggesting test-retest reliability as well as internal consistency. Moreover, Brumariu et al. (2018) concluded that the SS was related to other attachment measures (e.g. Inventory of Parent and Peer Attachment, IPPA, Armsden \& Greenberg, 1987; Coping Strategies Questionnaire Finnegan, Hodges, \& Perry, 1996; Child Attachment Interview (Shmueli-Goetz, Target, Fonagy, \& Datta, 2008). These relations did not appear to be moderated by child sex and/or age in the meta-analysis.

Most of the studies using the Kerns SS have accepted Kerns et al.'s original assumption that the scale measures a single construct (e.g. Dwyer, 2005; Marci et al., 2018; Van Ryzin \& Leve, 2012; Verschueren \& Marcoen, 2002, 2005), however some others have attempted to 
decompose the 15-item scale into subscales based on a priori assumptions (e.g. Lieberman et al., 1999) or on psychometric criteria (e.g. Barcsi et al., 2017). It is not clear, however, that the results of these studies improved the attachment-relevant utility of the Kerns et al.'s single-factor scale. For example, in the Barcsi et al. study, associations between a measure of children's depressive symptoms and the full-scale (15 items) or Reliance subscale (7 items) scores were not significantly different and were indistinguishable for any practical application purpose ( $r s=-.64$ vs. -.68 , respectively, for maternal scores and -.55 vs. -.59 , respectively, for father scores). Although the partitioning of the original item-set into subscales does not appear to have improved the measure as an attachment assessment, the fact that the items could be re-arranged on either logical or psychometric grounds prompted Kerns and associates (Kerns, Mathews, Koehn, Williams, \& Siener-Ciesla, 2015) to rethink the content and the intent of the original SS.

The original scale was intended to assess the secure base phenomenon during middle childhood and early adolescence (Kerns et al., 1996; Kerns, Tomich, \& Kim, 2006), however a self-study of the items suggested that the original item-set over-represented the safe haven aspect of the phenomenon (14 items) and under-represented the secure base for exploration aspect (1 item). Consequently, Kerns et al. (2015) added six items intended to measure secure base support for exploration (total of 7 items, 6 new). They suggested that the addition of these new items might afford possibilities of better assessing the attachment-relevant role of fathers, perhaps especially in middle-childhood and adolescence if fathers, more than mothers, provide support for challenging explorations of the physical and social environments (see Bögels \& Phares, 2008; Bretherton, 2010, for similar arguments).

In this study we investigated the factorial structure of the new SSQ (Kerns et al., 2015), considering children's perceptions of not only safe haven behaviors but also secure base support expected from caregivers. We included tests of measurement invariance across both mother-child and father-child relationships, taking into consideration the dependency of the observations and the ordinal nature of the data, using a multigroup analysis approach. Finally, we collected data in two countries (Portugal, USA) to test whether there was measurement invariance across these two cultural contexts.

This aspect of the study is an advance over previously published analyses of the psychometric properties of the SSQ and addresses a critical issue arising when a measure designed to assess attachment security in one socio-cultural milieu is imported by developmental scientists from a different socio-cultural milieu. That is, does the measure assess the same underlying construct in the same ways across cultural boundaries, even though attitudes, values, and goals concerning children, child-rearing, and parent-child relationships may differ across those boundaries. This issue has been raised by cultural psychologists with regard to the universality of attachment theory more generally (e.g. Keller, 2013; Rothbaum, Weisz, Pott, Miyake, \& Morelli, 2001), although the empirical evidence does tend to favor the Bowlby/Ainsworth position that infants and young children seek proximity and contact with primary caregivers and use them as a safe haven and secure base for exploration across socio-cultural boundaries (e.g. van IJzendoorn \& Sagi, 1999), contexts (e.g. Goossens \& van IJzendoorn, 1990; Howes, 1999; Vorria et al., 2003; Zeanah, Smyke, Koga, \& Carlson, 2005), and family types (e.g. Bokhorst et al., 2003; Juffer \& Rosenboom, 1997; van IJzendoorn et al., 2000).

In this study, two different socio-cultural groups were compared. One is an American sample, representing North American cultures that have been described as more 
individualistic (Triandis, 1995) and focused on independence, autonomy, assertiveness, and self-reliance (Hui \& Triandis, 1986). The other is a Portuguese Sample, representing Latin cultures, that have been characterized as endorsing more collectivist norms and values (Triandis, 1995) with emphases on conformity, compliance, respect for authority figures, and interdependent social relationships (Hui \& Triandis, 1986). The available evidence tends to favor a universalist position. For example, Posada et al. (2013), compared secure base behavior in young children as well as local experts' and parent's descriptions of optimal child behavior (using the Attachment Q-sort items) and found no substantive differences across several countries including Portugal and the USA. That is, the Q-profile of the hypothetically most secure child was positively and significantly correlated with the "optimal" profile across socio-cultural contexts. Of course, the facts that attachment security is defined similarly and that the profile of behaviors defined as typical of the very secure child in North America is significantly similar to the "optimal" profile across the socio-cultural groups assessed does not imply that the distributions of more and less secure infants and children will be the same across socio-cultural groups, nor are they expected to be identical within any socio-cultural group. Rather, it is the experience over time of the child within the context of her or his specific set of primary caregivers that is hypothesized to determine the relative security vs. insecurity of the attachment relationship.

This study also includes both parents as attachment figures. Although fathers are often viewed as secondary figures especially in middle childhood, empirical findings suggest that fathers, as well as mothers, serve as important attachment figures (Bowlby, 1982; Bretherton, 2010). Indeed, attachment theory recognizes that children may have a network of attachments, and that non-maternal attachments, including with fathers, are regular features of children's lives (e.g. Bureau, Deneault, \& Yurkowski, 2019; Lux \& Walper, 2019; Pinto, Veríssimo, Gatinho, Santos, \& Vaughn, 2015; Steele, Steele, \& Fonagy, 1996). However, the ways that attachment relationships with mother and father are established and maintained may differ qualitatively and it is possible that the domains of behavior, affect, and cognition influenced by attachment differ for mothers and fathers (e.g. Barcsi et al., 2017; Boldt, Kochanska, Grekin, \& Brock, 2016; Kerns et al., 2015).

Given the empirical evidence concerning the universality of attachment phenomena, we expected to find support for the two-factor structure (i.e. safe haven and secure base support for exploration) suggested by Kerns et al. (2015). Further, we expected the factor structure to demonstrate measurement invariance across mother-child and father-child relationships, between boys and girls, and across sociocultural boundaries (i.e. Portugal and the USA).

\section{Method}

\section{Participants}

\section{Portuguese sample}

Participants were 285 children (154 girls and 131 boys), ranging from 10 to 14 years old ( $M$ $=10.74, S D=.76)$, and $80 \%$ had siblings. They attended $5^{\text {th }}$ or $6^{\text {th }}$ grade from 3 Lisbon Schools (140 in the $5^{\text {th }}$ grade, and 145 in the $6^{\text {th }}$ grade). Parents reported their child's race/ ethnicity as $98 \%$ White/Caucasian and $2 \%$ for African Portuguese. Each class included an 
average of 20 children, and all children from each classroom were invited to participate. All the children assented to participate in the study. We limited our analyses to children who had data for both mother-child and father-child relationship because we wanted to make mother-father comparisons.

Most of the parents were either married or cohabiting (70\%). For mothers, $1.2 \%$ reported having a high school diploma or less, 38.3\% reported having an associate degree or some college, 55.3\% reported a having four-year degree, and $5 \%$ of mothers reported having 1-4 years of postgraduate education. For fathers, families reported that $5.5 \%$ had a high school diploma or less, $34.8 \%$ had an associate's degree or some college, 53.8\% had a four-year degree, and $5.9 \%$ reported 1-4 years of postgraduate education. Mothers' ages ranged from 29 to 60 years $(M=42.73, S D=4.68)$, and fathers' ages ranged from 29 to $69(M=44.98, S D=$ 5.45). More than $98 \%$ were full-time working parents (greater than or equal to $40 \mathrm{~h}$ per week).

\section{USA sample}

Participants were 172 children (70 girls and 102 boys) ranged from 9 to 14 years old $(M=$ $10.99, S D=1.02), 83.7 \%$ had siblings. One participant was in the third grade, one was in the $9^{\text {th }}$ grade, and the remainder were in $4^{\text {th }}-8^{\text {th }}$ grades $(M=5.59, S D=1.24)$. Parents reported their child's race/ethnicity as $79.7 \%$ White/Caucasian, $15.1 \%$ mixed race or other and less than 3\% each for African American, American Indian, Hispanic, or Asian. We recruited families via fliers distributed to local public and private schools and summer camps. One child discontinued responding to questions about his father and was excluded from the study. We limited analyses to children who had data for both motherchild and father-child relationships to make mother-father comparisons.

Families reported that $77.9 \%$ were intact (e.g. neither divorced nor separated). Regarding employment status, $57 \%$ of mothers and $85 \%$ of fathers worked at least fulltime hours (greater than or equal to $35 \mathrm{~h}$ per week). For mothers, $8.8 \%$ reported having a high school diploma or less, $14.6 \%$ reported having an associate degree or some college, $40.4 \%$ reported a having four-year degree, and $36.2 \%$ of mothers reported having 1-4 years of postgraduate education. For fathers, families reported that $21.3 \%$ had a high school diploma or less, $13.6 \%$ had an associate's degree or some college, $35.7 \%$ had a four-year degree, and $29.1 \%$ reported $1-4$ years of postgraduate education.

\section{Procedure}

\section{Portuguese sample}

Data were collected after the permission of the school authorities and after receiving the informed consent of families. All classroom assessments were performed during regular school hours, in class, during a single 45-min session. A research assistant was present to introduce the study and to answer questions. The instructions given emphasized the confidentiality of the data, the anonymous and voluntary participation, and the importance of completing the questionnaire individually.

\section{USA sample}

Children attended a lab visit with their parents at a Midwestern US institution. Visits typically lasted between 90 and 120 minutes. Each visit was coordinated by two research assistants (one graduate and one undergraduate student). During the visit, children 
completed the SSQ questionnaires about two parents (unless they were only in contact with one parent) in addition to other questionnaire measures and attachment tasks. Children with both biological and stepparents responded regarding the parent to whom they felt closest. Parents and children received \$25 each in compensation for their participation.

\section{Measures}

\section{Security Scale questionnaire (SSQ; Kerns et al., 2015)}

The SSQ measures the degree to which the child sees mother/father as responsive and available figure (Kerns et al., 2015). In the original version (Kerns et al., 2001) all 15 items were aggregated in a single dimension that measured attachment security to mother/ father. This new 21-item version (Kerns et al., 2015) is organized in a two factors structure. The Safe Haven Support (SHS) subscale (14 items from the original SS) evaluates open communication about needs and emotions and whether a child uses parents as a safe haven when experiencing distress, for example "going to a parent when upset". The newer Secure Base Support (SBS) subscale (6 new items plus one item from the original SS) evaluates parents' encouragement and support of exploration of novelty, for example "encouraging the child to be themselves or to try new things". Each item is rated on a 4-point scale using Harter's (1982) format "Some kids ... but ... Other kids", with higher scores indicating greater security. Specifically, for each question, the child is presented with two different types of children, and then must decide which is more similar to them, e.g. "Some kids wish their dad would help them more with their problems, BUT other kids think their dad helps them enough". After that, the child specifies whether they are "sort of like" or "really like" the child in the question. Each item is scored from 1 to 4, with a higher score representing greater security. In the original study (Kerns et al., 2015), SHS Cronbach's alphas were .88 for mother, and .90 for father, and .76 and .74 considering SBS for mother and father, respectively. In addition, in Kerns et al. (2015) the two subscales were significantly correlated with ratings of Safe Haven and Secure Base support scored from the Friends and Family Interview (Steele \& Steele, 2005).

The SSQ was translated from the original English version into Portuguese following the procedures outlined by "Committee Approach" (Brislin, 1980), a methodology for cultural adaptation of psychological questionnaires. A first version was then applied to a small group of children, to ensure that all items were understandable and thus suitable.

\section{Analytic plan}

To evaluate the model fit and its consistency with the data, we used the $R$ packages Lavaan (Rosseel, 2012), SemTools (Jorgensen, Pornprasertmanit, Schoemann, \& Rosseel, 2018) and Overlapping (Pastore, 2016) for Confirmatory Factor Analyses (CFA) and Measurement Invariance (MI) evaluation. First, we explored items distributions for child responses when referring to the maternal or to paternal relationship distinctly. Then, we performed CFA on items considering a two-factor construct, and given the ordinal nature of the data we used the Robust Weighted Least Squares (RWLS) estimator (Flora \& Curran, 2004). Configural invariance was evaluated using three model-fit indices: the Comparative Fit Index (CFI, representing a good fit if > .95, an acceptable fit when between .90 and .95, 
a poor fit if <.90), the Root Means Square Error Approximation (RMSEA, acceptable fit if < .06) (Hu \& Bentler, 1999), and the Weighted Root Mean Square Residual (WRMR good when less than 1.0, with lower values indicating better fit (Yu, 2002; Yu \& Muthen, 2002). When model indices were not adequate we took the factor loadings into consideration to decide whether to maintain or exclude the items (Hair, Anderson, Tatham, \& Black, 1998). Based on final model, scale reliability was evaluated using ordinal Cronbach's alpha.

We explored measurement invariance across both mother-child and father-child relationships, considering the dependency of the observations (same child reporting for relationship with mother and father) by correlating the residual variances of the same indicator across mother and father. We used Intra-class Correlation Coefficients (ICC, consistency version, corresponding to a one-way random effects model, or ICC $[1,1]$; Shrout \& Fleiss, 1979) to evaluate associations between mother-child and father-child attachment. We used the bootstrap procedure, which allows us to estimate the sampling distribution of factorial loadings, resampling with replacement from the original sample without normality assumption (Efron \& Tibshirani, 1993). We extracted the empirical distribution of each factor loading by conducting 4000 bootstrapped replication samples from the original data and analyzing the overlapping area for each corresponding pair of items (e.g. item 1 for mother, and item 1 for father). Factor loading distributions and the proportion of overlap across paired items (mother and father) were graphically represented and analyzed. Values higher than .75 were considered excellent, $>.60$ good, $>.40$ fair, and less than .40 poor, as recommended by Cicchetti (1994).

Sex invariance was tested using a multi-group confirmatory factor analysis (MG-CFA), a weighted least square means (WLSM) and variance-adjusted estimator (WLSMV) with theta parameterization to take into account the ordinal nature of the items (Brown, 2006). If the difference in the fit indices ( $\triangle \mathrm{CFI}$ and $\triangle \mathrm{RMSEA}$ ) between a model and the (preceding) less constrained model was equal or less than .01 for $\triangle C F I$, and equal or less than .015 for $\triangle \mathrm{RMSEA}$, we considered that the level of measurement invariance was achieved (Chen, 2007). If a model's fit was poorer (i.e. $\Delta C F I>.01$ ), we identified the non-invariant item(s) by considering the modification indices and then removed the corresponding equality constraint between the two groups (i.e. the parameter was freely estimated in each group). If the differences between the CFI and RMSEA in the resulting and the less constrained models exceeded the accepted cutoffs, partial invariance was achieved, and the parameter remained unconstrained in the subsequent models of the measurement invariance assessment process. Using the final model, we performed a multi-level analysis to explore possible differences between samples (PT vs. USA). Finally, we evaluate potential differences in attachment security and performed a multivariate analysis with child sex and age as predictors.

\section{Results}

As we can see in Table 1, most of the items presented a left-skewed distribution (as expected in a non-clinical sample as ours). We took the ordinal nature of the data into account and used the DWLS robust estimator (RWLS) (Flora \& Curran, 2004).

As we can see in Table 2, the initial model (M) testing all 21 items organized as one factor presented a good CFI (.95), although RMSEA was a little higher than .06 and WRMR higher than 1. In the next model (M1), we established a two-factor structure. Although this 


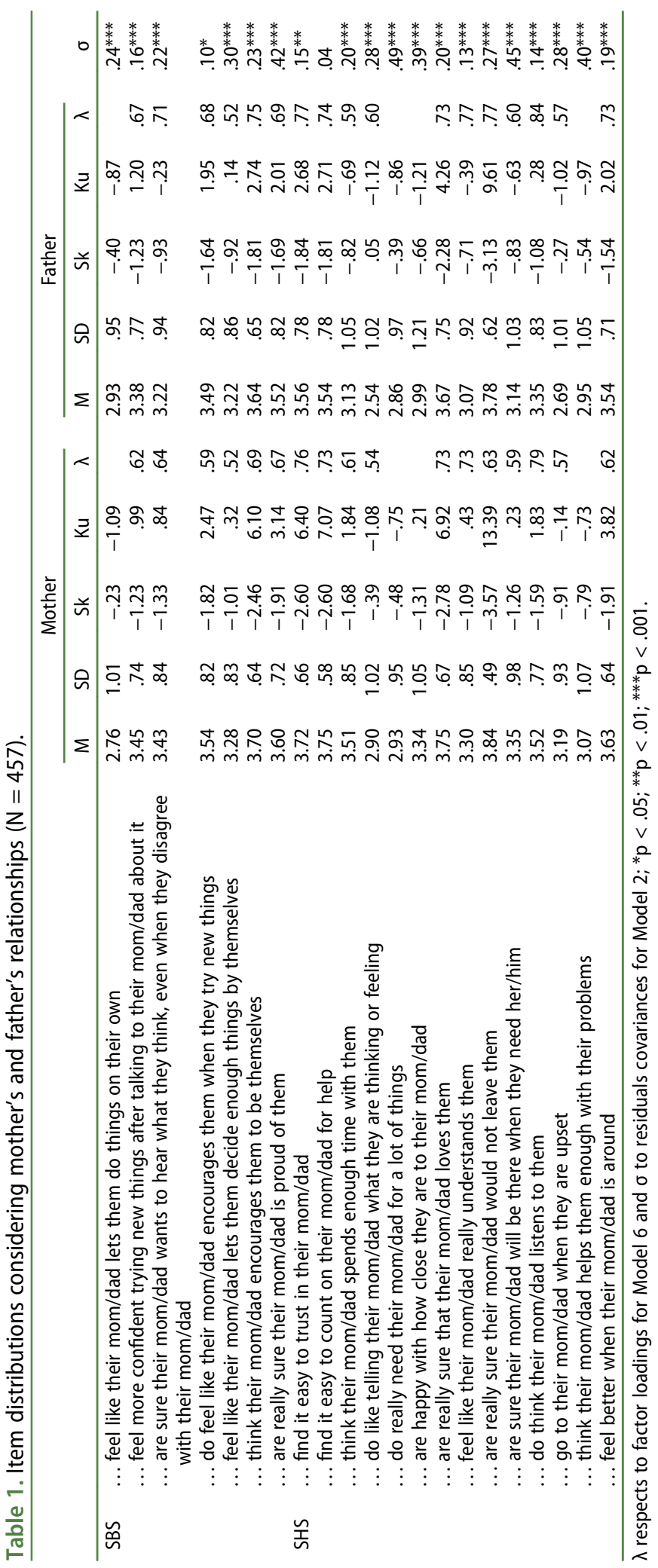


Table 2. Fit indices for the CFA models of the SSQ.

\begin{tabular}{lccccccc}
\hline Model & Item deleted & RWLS & $\mathrm{df}$ & $\mathrm{p}$ & $\mathrm{CFI}$ & RMSEA [90\% CI] & WRMR \\
\hline M & & 2086.59 & 818 & $<.001$ & .95 & $.069[.066 ; .072]$ & 1.62 \\
M1 & & 2038.82 & 813 & $<.001$ & .95 & $.068[.065 ; .071]$ & 1.59 \\
M2 & 1508.20 & 792 & $<.001$ & .97 & $.049[.045 ; .053]$ & 1.30 \\
M3 & 7 & 1393.50 & 714 & $<.001$ & .98 & $.049[.046 ; .053]$ & 1.29 \\
M4 & 2 and 7 & 1278.62 & 640 & $<.001$ & .98 & $.049[.045 ; .053]$ & 1.28 \\
M5 & 2,7 and 19 & 1003.63 & 570 & $<.001$ & .99 & $.039[.034 ; .043]$ & 1.14 \\
M6 & $2,7,19$ and 9 & 834.80 & 504 & $<.001$ & .99 & $.032[.027 ; .037]$ & 1.06 \\
\hline
\end{tabular}

$\mathrm{N}=$ 457. $\mathrm{M}=$ one dimension Model; $\mathrm{M} 1$ = Two dimensions without residual covariances; $\mathrm{M} 2$ = Two dimensions with residual covariances between mother's and father's items. RWLS = Robust Weighted Least Squares; CFI = Comparative Fit Index; RMSEA = Root Mean Square of Approximation; WRMR = Weighted Root Mean Square Residual.

modification did not change the model fit, we kept it for theoretical reasons. In the following step (M2), we added residual covariances between mothers and fathers related items (since it was the same child responding for both parents), which lead to an improvement, with most of the indicators presenting good values. However, considering that WRMR was still higher than 1, we gradually eliminated four items that presented lower factor loadings $(\lambda<.50)$ for either or both father and mother. In M3, we dropped item 7, "Some kids do not really need their dad/mom for much/Other kids need their dad/ mom for a lot of things" $(M=2.93, S D=.95, \lambda=.37$ for mother, and $M=2.86, S D=.97, \lambda=$ .21 for father, also the residual covariance was .49; $\rho<.001)$. In M4, we removed item 2, "Some kids feel like their dad/mom butts in a lot when they are trying to do things/Other kids feel like their dad/mom lets them do things on their own" $(M=2.76, S D=1.01, \lambda=.43$ for mother, and $M=2.93, S D=.95, \lambda=.34$ for father; residual covariance was .24; $\rho<.001)$. In M5, we dropped item 19, "Some kids wish their dad/mom would help them more with their problems/Other kids think their dad/mom helps them enough $(M=3.07, S D=1.07, \lambda=.44$ for mother, and $M=2.95, S D=1.05, \lambda=.51$ for father, residual covariance was $.40 ; \rho<.001)$. In M6, we deleted item 9, "Some kids wish they were closer to their dad/mom/Other kids are happy with how close they are to their dad $/ \mathrm{mom}(M=3.34, S D=1.05, \lambda=.49$ for mother, and $M=2.99, S D=1.21, \lambda=.55$ for father, residual covariance was .39; $\rho<.001$ ). The model (M6) presented a good fit (CFI $=.99, \mathrm{RMSEA}=.03, \mathrm{WRMR}=1.06)$.

Initial reliability indices were good for both the mother-child and father-child relationship. For mothers, the ordinal Cronbach's alphas were .79 for SBS and .89 for SHS (ordinal omegas were .79, and .89, respectively). For fathers, Cronbach's alphas were .83 for SBS and .91 for SHS (ordinal omegas .83, and .91, respectively). These results were similar to the Cronbach alphas obtained by Kerns et al. (2015) in the original study: SBS alphas .76 and .74 for mother and father, respectively, and SHS alphas .88 and .90 for mother and father, respectively.

To test for measurement invariance across mother-child and father-child relationships, we used model 6 (that already correlates the residual variances of the same indicator across mother and father), and estimated the empirical distribution of the bootstrapped standardized loadings and the overlapping area for each pair of items (Figure 1). Overall, the bootstrapped distributions were approximately normal. For most of the items the overlapping area was high, suggesting construct invariance when comparing children's report on mothers and fathers as SBS and SHS, except for two items which overlapped less than $30 \%$ : item 13, "Some kids are really sure their dad/mom would not leave them ... " $(M=3.84$, $S D=.49, \lambda=.63$ for mother, and $M=3.78, S D=.62, \lambda=.77$ for father, residual covariance 

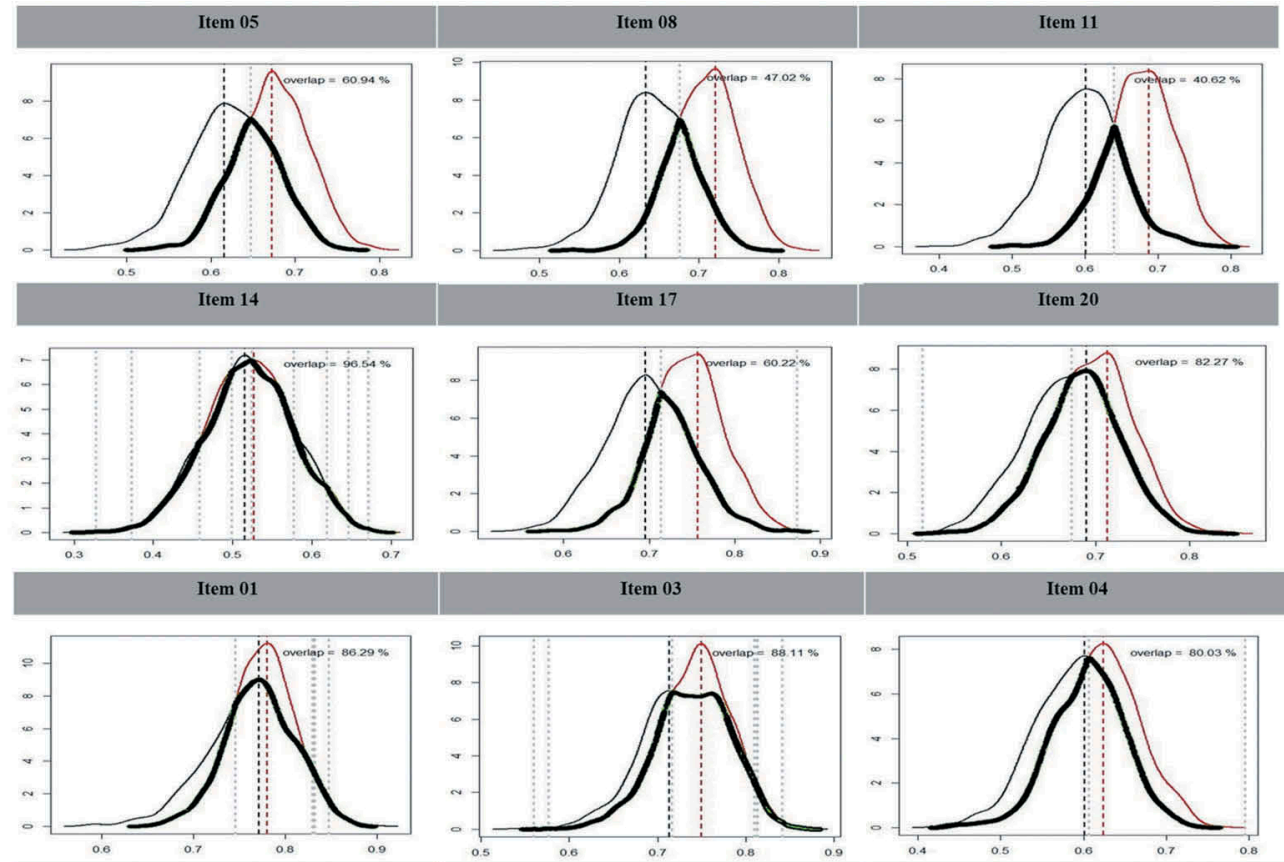

Item 06
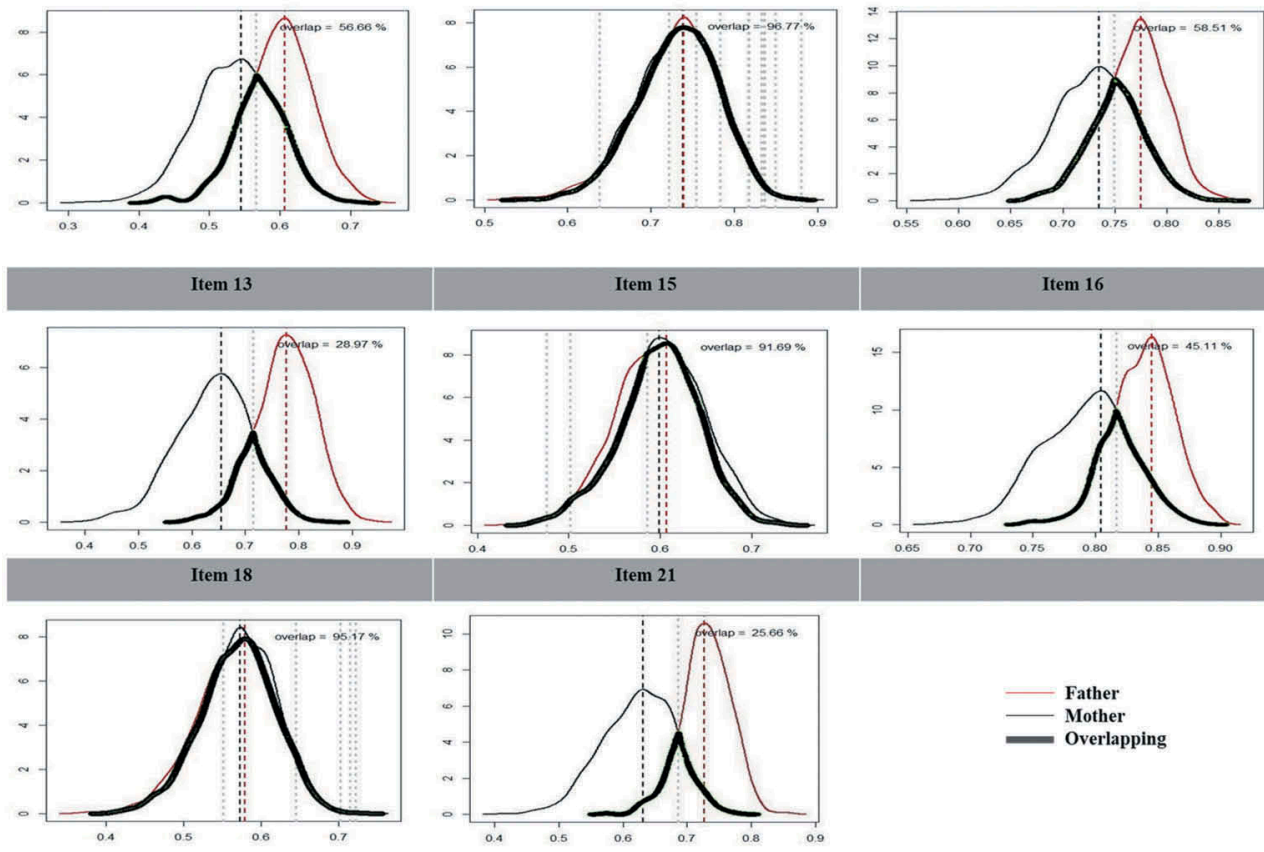

Figure 1. Distribution of the bootstrapped standardized factor loadings for mother and father items and their overlapping area.

was .27, $\rho<.001$, and overlapping area was 28.97\%), and item 21, "Some kids feel better when their dad/mom is around ..." ( $M=3.63, S D=.64, \lambda=.62$ for mother, and $M=3.54, S D=.71$, $\lambda=.73$ for father, residual covariance was .19, $\rho<.001$, and overlapping area was $25.66 \%$ ). 
Further, factor loading distributions across SBS were very similar (see Table 1), specifically, for both mother' and father' related items, the best loading was for item 17, "Some kids think their dad/mom encourages them to be themselves ..." $(M=3.70, S D=.64, \lambda=.69$ for mother, and $M=3.64, S D=.65, \lambda=.75$ for father, with residual covariance of $.23, \rho<.001$, and overlapping area was 60.22\%), and the lowest loading was for item 14, "Some kids feel like their dad/mom lets them decide enough things by themselves ..." $(M=3.28, S D=.83, \lambda=.52$ for mother, and $M=3.22, S D=.86, \lambda=.52$ for father, residual covariance was $.30, \rho<.001$, and overlapping area was 96.54\%). For SHS, item 16, "Some kids think their dad/mom does not listen to them ..." $(M=3.52, S D=.77, \lambda=.79$ for mother, and $M=3.35, S D=.83, \lambda=.84$ for father, residual covariance was .14, $\rho<.001$, and overlapping area was $45.11 \%$ ) had the highest factor loadings for both mothers and fathers, and item 6, "Some kids do not really like telling their dad/mom what they are thinking or feeling- ..." $(M=2.90, S D=1.02, \lambda=.54$ for mother, and $M=2.54, S D=1.02, \lambda=.60$ for father, residual covariance was $.28, \rho<.001$, and overlapping area was 56.66\%) had the lowest factor loadings for both mothers and fathers. The final model (M7) presented a good fit $(C F I=.99, \mathrm{RMSEA}=.03, \mathrm{WRMR}=1.03)$.

Table 3 shows descriptive values for SBS and SHS by sex and country. For the test of invariance across sex, we used model 7 and performed a multi-group confirmatory factor analysis (MG-CFA). Since the results were below the cut point $(\triangle C F I=.003$, RMSEA $=-.007)$, metric invariance was achieved. Similarly, results also suggested scalar invariance $(\triangle C F I=-.004, \triangle R M S E A=.009)$, suggesting that differences in the means between those two groups (boys and girls) reflect differences in the underlying latent trait rather than in the measure. We also performed a multilevel analysis to explore possible differences between samples (PT vs. USA), no statistic differences were found (see Table 4). For that reason, we treat the sample as one in subsequent analyses.

Using the final model (model 7), we analyze SSQ factors for both parents. SBS and SHS factors were highly and positively correlated within a relationship for both mothers and fathers ( $r=.72, \mathrm{p}<.001$, and $r=.75, \mathrm{p}<.001$, respectively). This is similar to the magnitude of association as reported by Kerns et al. (2015), although information was available only for mother's relationship $(r=.75)$. We also examined mother-father correlations for SBS and SHS, and we found there was a positive and significant correlation for SBS $(r=.44, p<.001)$, and SHS $(r=.41, p<.001)$. Children reported higher levels of SBS with mothers than with fathers (mother $M=3.50, S D=.47$, and father $M=3.41, S D=.54, t(456)=3.66, p<.001$ ), though the

Table 3. Descriptive values for SBS, SHS and age considering sex and countries.

\begin{tabular}{|c|c|c|c|c|c|c|c|}
\hline & & \multirow[b]{3}{*}{$\mathrm{N}$} & \multirow[b]{2}{*}{ Age } & \multicolumn{2}{|c|}{ SBS } & \multicolumn{2}{|c|}{ SHS } \\
\hline & & & & Mother & Father & Mother & Father \\
\hline & & & $M(S D)$ & $M(S D)$ & $M(S D)$ & $M(S D)$ & $M(S D)$ \\
\hline \multirow[t]{3}{*}{ PT } & Male & 131 & $10.79(.80)$ & $3.51(.51)$ & $3.42(.57)$ & $3.49(.50)$ & $3.23(.61)$ \\
\hline & Female & 154 & $10.71(.71)$ & $3.63(.40)$ & $3.50(.47)$ & $3.53(.46)$ & $3.25(.50)$ \\
\hline & Total & 285 & $10.74(.76)$ & $3.58(.45)$ & $3.46(.52)$ & $3.51(.48)$ & $3.24(.55)$ \\
\hline \multirow[t]{3}{*}{ US } & Male & 102 & $11.13(1.29)$ & 3.33 (.49) & $3.35(.55)$ & $3.31(.48)$ & $3.16(.61)$ \\
\hline & Female & 70 & $10.80(1.41)$ & $3.44(.42)$ & 3.27 (.59) & $3.34(.56)$ & $3.00(.67)$ \\
\hline & Total & 172 & $10.99(1.34)$ & 3.38 (.47) & $3.32(.57)$ & $3.32(.50)$ & $3.10(.64)$ \\
\hline \multirow[t]{3}{*}{ Total } & Male & 233 & $10.94(1.06)$ & $3.43(.51)$ & $3.39(.56)$ & 3.41 (.49) & $3.20(.61)$ \\
\hline & Female & 224 & $10.74(.98)$ & $3.57(.41)$ & $3.43(.52)$ & $3.47(.50)$ & $3.17(.57)$ \\
\hline & Total & 457 & $10.84(1.02)$ & $3.50(.47)$ & $3.41(.54)$ & $3.44(.49)$ & $3.19(.59)$ \\
\hline
\end{tabular}

${ }^{*} \mathrm{p}<.05 ;{ }^{* *} \mathrm{p}<.01 ;{ }^{* * *} \mathrm{p}<.001$. 
Table 4. Parameter estimates (and standard errors) for SBS and SHS.

\begin{tabular}{|c|c|c|c|c|c|c|c|c|}
\hline \multirow[b]{3}{*}{ Fixed Coefficients } & \multicolumn{4}{|c|}{ SBS } & \multicolumn{4}{|c|}{ SHS } \\
\hline & \multicolumn{2}{|c|}{ Mother } & \multicolumn{2}{|c|}{ Father } & \multicolumn{2}{|c|}{ Mother } & \multicolumn{2}{|c|}{ Father } \\
\hline & & & & & & & & \\
\hline Intercept & $3.43^{* * *}$ & $(.07)$ & $3.39^{* * * *}$ & $(.05)$ & $3.40^{* * *}$ & $(.07)$ & $3.20^{* * *}$ & (.28) \\
\hline Age & -.04 & $(.02)$ & $-.07^{* *}$ & $(.02)$ & -.01 & (.02) & $-.06^{*}$ & $(.03)$ \\
\hline Sex & $.11^{* *}$ & (.04) & .01 & $(.05)$ & .04 & (.05) & -.05 & (.05) \\
\hline Variance Components & & & & & & & & \\
\hline Between Countries & .01 & $(.01)$ & .00 & $(.00)$ & .01 & $(.01)$ & .00 & $(.00)$ \\
\hline
\end{tabular}

${ }^{*} \mathrm{p}<.05 ;{ }^{* *} \mathrm{p}<.01 ;{ }^{* * *} \mathrm{p}<.001$.

magnitude of the difference is considered small (Cohen's $d=.18$ ). In addition, they also reported higher SHS for mothers (mother $M=3.44, S D=.49$; father $M=3.19, S D=.59, t$ $(456)=9.22, p<.001)$, with a medium effect size (Cohen's $d=.46$ ).

Finally, to evaluate the impact of sex and age on SSQ dimensions, a multivariate analysis was performed. Both SBS and SHS in father-child relationships were found to be significantly lower in older children $(\beta=-.07, p<.01$, and $\beta=-.06, p<.05$, respectively). When exploring the impact of child sex, we found that SBS was significantly higher for mother-daughter relationships $(\beta=.11, p<.01)$.

\section{Discussion}

The first goal of this study was to examine the structure of the recently revised Kerns SSQ with a statistical approach that considered both the ordinal nature of the data and the dependency of the observations, which arise from the fact that each child contributed SSQ scores for both parents (Table 1). We compared single and two-factor solutions to determine whether adding secure base support content to the original allowed for a broader understanding of the secure base phenomenon in middle childhood. In all models tested, mother and father data were analyzed simultaneously. The initial analyses (models M and M1 in Table 2), fit indices showed no significant differences between the single and two-factor solutions, which is not surprising because the factors are expected to be significantly correlated as they reflect two aspects of the same (e.g. secure base) phenomenon. However, when the residualized covariances for the items were included in the two-factor solution (model M2 in Table 2), model fit improved, supporting our decision to consider that the same child is reporting on both mother and father. Model fit was further improved after deletion of four items (model M6 in Table 2) that had relatively low (compared to other items) associations with the general security factor. We note that these items have been deleted by other researchers when factor analyzing the original 15-item SSQ (e.g. Bacro, 2011; Barcsi et al., 2017; Kerns \& Mathews, 2010; Marci et al., 2018). Measures of internal consistency (ordinal Cronbach alphas) were nearly identical for both mothers and fathers across the two subscales.

Even though the single and dual-factor solutions were indistinguishable in terms of model fit criteria, there are theoretical reasons for preferring the two-factor solution. The secure base phenomenon concept was initially construed in terms of two related processes, namely as secure base support (SBS) for exploration and as safe haven support (SHS) when the child was threatened or otherwise distressed (see Waters \& Cummings, 2000). In 
the context of the revised Kerns SSQ, children's beliefs concerning the availability, responsiveness, and reliance on parents are believed to be indicators of their representations of SHS processes and their beliefs related to the encouragement and support when exploring novelty are indicators of SBS processes. Kerns et al. (2015) argued that safe haven beliefs are important in the child's management of distressing situations when attachment figure(s) are not immediately present, while secure base support beliefs are important supports for confidence in exploring novel physical, social, and emotional domains in the absence of an attachment figure. In these data, SBS ad SHS scores were somewhat different in their relation to child sex (girls reported more SHS with mothers) and in the magnitude of mother-father differences (the differences were stronger for reports of SHS), suggesting it may be useful to examine SBS and SHS scales separately.

As expected, the two security dimensions were positively associated within both mother and father datasets. That is, children reporting higher levels of SBS also tended to report higher levels of SHS. Kerns et al. (2015) reported similar results. Further, analyses relating each dimension across mothers and fathers were positive and significant, suggesting that children, on average, tend to view SBS and SHS in a similar manner, but not necessarily identical, across both parents. Even so, children's scores on both secure base and safe haven dimensions were higher when they described their mothers than when they described their fathers, and girls (only) also reported higher values for SBS when referring to their mothers.

Studies with the original version of the Security Scale have yielded mixed results regarding parent or child sex differences in attachment security. Some studies found that children reported greater security when characterizing their mothers than when characterizing their fathers (Barcsi et al., 2017). Other studies found that this discrepancy between parents depended on child gender. In two studies, girls and boys reported greater security for the same-sex parent (Diener, Isabella, Behunin, \& Wong, 2008; Sarracino, Presaghi, Degni, \& Innamorati, 2011), and in another study, girls reported greater security in relationships with their mothers (Kerns et al., 2006). Another study did not find gender differences in security of attachment to mothers and fathers (Doyle, Markiewicz, Brengden, Lieberman, \& Voss, 2000). Our finding for the safe haven scale is similar to results presented in previous studies. Kerns et al. (2015) assessed both SHS and SBS separately. Consistent with our findings, children in their study reported greater safe haven support from mothers. However, they also reported a non-significant trend for children to report greater secure base support from fathers, which is not consistent with our findings. Given the mixed results reported in the literature, we are not inclined to treat sex differences in these scores as meaningful from the perspective of attachment theory. More important is the consistent finding that both, boys and girls, organize secure base behavior around all of their primary caregivers, at least when each caregiver acts as a secure base by providing appropriate supports over a significant period of time. Inevitably there will be some intra-individual differences for SBS and SHS support, but it is not clear that these should be differentiated by parental role, nor is it clear that differences in the balance of SBS and SHS support should lead to different attachment representations for one caregiver or another.

We also examined these data for age differences in the SBS and SHS scale scores, given that the range of ages spans the transition from middle childhood to early adolescence. In this sample, younger children tended to describe their fathers as more supportive for both 
the SHS and SBS aspects of the secure base phenomenon than did older children. Similar findings for age differences were reported by Verschueren and Marcoen (2005) with younger children describing their relationship with fathers more positively with regard to SHS (using the original Kerns SSK) than did older children, and girls being more positive than boys. We do not interpret these age changes as reductions in the quality of the father/child relationship but rather as an age-appropriate adjustment with respect to the kinds of challenges that require the availability of the secure base. That is to say, many problems/challenges that might have prompted proximity and help-seeking in a younger child can be managed effectively by an adolescent. Thus, the adolescent may seek help/ guidance less often but remain confident that help would be available if needed and requested.

The second major goal of this study was to assess the measurement invariance for the revised Kerns SSQ with regard to sex of parent and with regard to country of origin. Our results show that the SSQ can be used to study both child/mother and child/father attachment representations because the factorial structure (based on Model 6 with 17 items) was substantially invariant. However, we note that the overlapping procedure highlighted two items (i.e. "sure that wouldn't be left", and, "feeling better when parents are around") as presenting significantly different factor loadings for fathers and mothers. Those items seem to better reflect SHS for fathers than for mothers, a finding that is similar to previous studies (see Bacro, 2011; Marci et al., 2018). In addition, results reported in Table 4 suggest that the variance in the SHS and SBS scores for mothers and fathers that can be attributed to the country of origin is trivially small. That result suggests that the scale scores for the revised Kerns SSQ are not influenced in a significant way by the country of origin.

As discussed above, establishing the invariance of measurements is a critical step in cross-cultural research on attachment (indeed, for any instrument measuring constructs from the developmental, behavioral, and social sciences that is used across different cultures). The results we obtained give confidence that we can interpret the findings from the revised SSQ similarly for both samples and this confidence generalizes to similar samples from these countries. Without establishing measurement invariance, it would not be possible to interpret significant between-group differences (or similarities) that might be observed. We note that these psychometric considerations often are not tested explicitly in cross-cultural social science research. Rather, investigators usually assume that successful back-translation of items is sufficient to assume measurement invariance. We also note that measurement invariance does not imply that developmental phenomena, including attachment formation and maintenance, follow the same developmental schedule or lead to the same outcomes over the lifespan in every culture. Whether or not developmental phenomena are adaptations that are applicable to all human societies, without regard to local culture is a hypothesis that must be tested in the relevant populations before it can be accepted or rejected. With regard to attachment development and its implications for subsequent emotional, social, and personality, the available evidence is consistent with the hypothesis of universality.

In conclusion, our findings suggest that the Portuguese 17-item version of the SSQ can be considered a capable and cost-effective tool to measure perceived attachment security in middle childhood for both mother and father relationships in Portuguese samples. Some limitations of the study suggest directions for future research. Although relatively 
simple to use, quick to administer, and helpful to provide a first impression of relationship quality, self-report questionnaires of attachment are limited. Even when reports are internally consistent, they are not equivalent to narrative representational measures of attachment. For example, self-report measures generally reflect judgements on items that are aggregated to form linear scales that must be taken at face value by the investigator while narrative measures are scored by trained coders who identify specific patterns in the narratives that lead to qualitative judgments about attachment quality (secure vs. insecure) rather than quantitative trait-like judgements about the amount (or frequency) of attachment relevant dimensions (e.g. security). Nevertheless, children's reports of attachment obtained by both questionnaire and narrative assessments are associated with many outcomes implied by attachment theory (Brumariu et al., 2018) and neither can be dismissed out of hand. We also need to replicate the study in a larger sample that is more representative of the Portuguese population. It will also be useful in future research to include multiple assessment methods for attachment and other constructs as well as multiple sources of information (e.g. parents, teachers) to assess the convergent, concurrent and predictive validity of the SSQ.

Finally, although moderately strong correlations between mother and father dimensions were found in this study, there are children with different representations for each parent. When and if an integration of different attachment representations in a unique model happens is still an important and open question in attachment research. In order to address this point, we intend in future studies, to study participants repeatedly over time to better understand stability and integration of SBS and SHS, and further evaluate the utility of distinguishing these two types of support.

\section{Acknowledgments}

The authors are grateful for the participation and support from students and teachers who have welcomed researchers into their classrooms. The authors thank all the members of the research team for their assistance and support.

\section{Disclosure statement}

No potential conflict of interest was reported by the authors.

\section{Funding}

This work was supported by the Portuguese Foundation for Science and Technology (FCT) [UID/PSI/ 04810/2019].

\section{ORCID}

Manuela Veríssimo (D) http://orcid.org/0000-0003-4356-5696

António J. Santos (D) http://orcid.org/0000-0003-2451-9352

Brian E. Vaughn (ID http://orcid.org/0000-0002-0035-2976

Marissa Gastelle (ID http://orcid.org/0000-0002-2095-5221

Kathryn A. Kerns (D) http://orcid.org/0000-0001-9579-0188 


\section{References}

Ainsworth, M. (1989). Attachment beyond infancy. American Psychologist, 44(4), 709-716.

Ainsworth, M., Blehar, M., Waters, W., \& Wall, S. (1978). Patterns of attachment: A psychological study of the strange situation. Hillsdale, NJ: Erlbaum.

Al-Yagon, M. (2011). Adolescents' subtypes of attachment security with fathers and mothers and self-perceptions of socioemotional adjustment. Psychology, 2(4), 291-299.

Armsden, G. C., \& Greenberg, M. T. (1987). The inventory of parent and peer attachment: Individual differences and their relationship to psychological well-being in adolescence. Journal of Youth and Adolescence, 16(5), 427-454.

Bacro, F. (2011). Validation francophone de l'échelle de sécurité des perceptions d'attachement au père et à la mère (Kerns, Klepac \& Cole, 1996) [French validation of the child-father and childmother attachment perceptions security scale (Kerns, Klepac \& Cole, 1996)]. Revue européenne de psychologie appliquée, 6, 213-221.

Barcsi, B., Hollody, K., Peley, B., Dorn, K., Kerns, K., Rozsa, S., ... Kallai, J. (2017). Security, reliance and availability: Psychometric features of the Kerns' Security Scale in Hungarian population. Mentálhigiéné És Pszichoszomatika, 18(1), 1-23.

Bögels, S., \& Phares, V. (2008). Fathers' role in the etiology, prevention and treatment of child anxiety: A review and new model. Clinical Psychological Review, 28(4), 539-558.

Bokhorst, C. L., Bakermans-Kranenburg, M. J., Fearon, R. M., van IJzendoorn, M. H., Fonagy, P., \& Schuengel, C. (2003). The importance of shared environment in mother-infant attachment security: A behavioral genetic study. Child Development, 74(6), 1769-1782.

Boldt, L., Kochanska, G., Grekin, R., \& Brock, R. L. (2016). Attachment in middle childhood: Predictors, correlates, and implications for adaptation. Attachment \& Human Development, 18(2), 115-140.

Bowlby, J. (1982). Attachment and loss (Vol. 1: Attachment). New York: Basic Books.

Bretherton, I. (2010). Fathers in attachment theory and research: A review. Early Child Development and Care, 180(1-2), 9-23.

Bretherton, I., Ridgeway, D., \& Cassidy, J. (1990). Assessing internal working models of the attachment relationship: An attachment story completion task for 3-year-olds. In M. T. Greenberg, D. Cicchetti, \& E. M. Cummings (Eds.), Attachment in the preschool years (pp. 273-308). Chicago: University of Chicago Press.

Brislin, R. (1980). Translation and content analysis for oral and written material. In H. Triandis \& J. Berry (Eds.), Handbook of cross-cultural psychology (Vol. 2, pp. 389-444). Needham Heights, MA: Allyn and Bacon.

Brown, T. (2006). Confirmatory factor analysis for applied research (1st ed.). New York: The Guilford Press.

Brumariu, L. E., Madigan, S., Giuseppone, K. R., Abtahi, M. M., \& Kerns, K. (2018). The Security Scale as a measure of attachment: Meta-analytic evidence of validity. Attachment \& Human Development, 20(6), 600-625.

Bureau, J.-F., Deneault, -A.-A., \& Yurkowski, K. (2019). Preschool father-child attachment and its relation to self-reported child socioemotional adaptation in middle childhood. Attachment \& Human Development. doi:10.1080/14616734.2019.1589065

Chen, F. F. (2007). Sensitivity of goodness of fit indexes to lack of measurement invariance. Structural Equation Modeling: A Multidisciplinary Journal, 14(3), 464-504.

Cicchetti, D. V. (1994). Guidelines, criteria, and rules of thumb for evaluating normed and standardized assessment instruments in psychology. Psychological Assessment, 6(4), 284-290.

Diener, M. L., Isabella, R. A., Behunin, M. G., \& Wong, M. S. (2008). Attachment to mothers and fathers during middle childhood: Associations with child gender, grade, and competence. Social Development, 17(1), 84-101.

Doyle, A. B., Markiewicz, D., Brengden, M., Lieberman, M., \& Voss, K. (2000). Child attachment security and self-concept: Associations with mother and father attachment style and marital quality. Merrill-Palmer Quarterly, 46(3), 514-539.

Dwyer, K. (2005). The meaning and measurement of attachment in middle and late childhood. Human Development, 48(3), 155-182. 
Efron, B., \& Tibshirani, R. J. (1993). An introduction to the bootstrap. New York: Chapman \& Hall.

Finnegan, R. A., Hodges, E. V. E., \& Perry, D. G. (1996). Preoccupied and avoidant coping during middle childhood. Child Development, 67(4), 1318-1328.

Flora, D., \& Curran, P. (2004). An empirical evaluation of alternative methods of estimation for confirmatory factor analysis with ordinal data. Psychological Methods, 9, 466-491.

Fraley, R. C. (2002). Attachment stability from infancy to adulthood: Meta-analysis and dynamic modeling of developmental mechanisms. Personality and Social Psychology Review, 6(2), 123-151.

George, C., Kaplan, N., \& Main, M. (1996). The adult attachment interview. Berkeley: University of California, Berkeley.

Goossens, F. A., \& van IJzendoorn, M. H. (1990). Quality of infants' attachments to professional caregivers: Relation to infant-parent attachment and day-care characteristics. Child Development, 61(3), 832-837.

Granot, D., \& Mayseless, O. (2001). Attachment security and adjustment to school in middle childhood. International Journal of Behavioral Development, 25(6), 530-541.

Guttmann-Steinmettz, S., Shoshani, A., Farhan, K., Aliman, M., \& Hirschberger, G. (2012). Living in the crossfire: Effects of exposure to political violence on Palestinian and Israeli mothers and children. International Journal of Behavioral Development, 36(1), 71-78.

Hair, J. F., Anderson, R. E., Tatham, R. L., \& Black, W. C. (1998). Multivariate data analysis (5th ed.). Englewood Cliffs, NJ: Prentice-Hall.

Harter, S. (1982). Perceived competence scale for children. Child Development, 53, 87-97.

Howes, C. (1999). Attachment relationships in the context of multiple caregivers. In J. Cassidy \& P. R. Shaver (Eds.), Handbook of attachment: Theory, research, and clinical applications (pp. 671-687). New York, NY: The Guilford Press.

Hu, L., \& Bentler, P. M. (1999). Cutoff criteria for fit indexes in covariance structure analysis: Conventional criteria versus new alternatives. Structural Equation Modelling: A Multidisciplinary Journal, 6(1), 1-55.

Hui, C. H., \& Triandis, H. C. (1986). Individualism-collectivism: A study of cross-cultural researchers. Journal of Cross-cultural Psychology, 17(2), 225-248.

Jorgensen, T. D., Pornprasertmanit, S., Schoemann, A. M., \& Rosseel, Y. (2018). semTools: Useful tools for structural equation modeling. R package version 0.5-1. Retrieved from https://CRAN.R-project. org/package=semTools

Juffer, F., \& Rosenboom, L. G. (1997). Infant-mother attachment of internationally adopted children in the Netherlands. International Journal of Behavioral Development, 20(1), 93-107.

Keller, H. (2013). Attachment and culture. Journal of Cross-cultural Psychology, 44, 175-194.

Kerns, K. A., Aspelmeier, J. E., Gentzler, A. L., \& Grabill, C. M. (2001). Parent-Child attachment and monitoring in middle childhood. Journal of Family Psychology, 15(1), 69-81.

Kerns, K. A., Brumariu, L. E., \& Seibert, A. (2011). Multi-method assessment of mother-child attachment: Links to parenting and child depressive symptoms in middle childhood. Attachment \& Human Development, 13(4), 315-333.

Kerns, K. A., Klepac, L., \& Cole, A. (1996). Peer relationships and preadolescents' perceptions of security in the child-mother relationship. Developmental Psychology, 32, 457-466.

Kerns, K. A., \& Mathews, B. (2010). Proposed revision for the security scale. Paper presented at the International Association of Relationship Research Conference, Herzlia, Israel.

Kerns, K. A., Mathews, B. L., Koehn, J. A., Williams, C. T., \& Siener-Ciesla, S. (2015). Assessing both safe haven and secure base support in parent-Child relationships. Attachment \& Human Development, 17, 337-353.

Kerns, K. A., Schlegelmilch, A., Morgan, T. A., \& Abraham, M. M. (2005). Assessing attachment in middle childhood. In K. A. Kerns \& R. A. Richardson (Eds.), Attachment in middle childhood (pp. 46-70). NY: Guilford.

Kerns, K. A., Tomich, P. L., \& Kim, P. (2006). Normative trends in children's perceptions of availability and utilization of attachment figures in middle childhood. Social Development, 15, 1-22.

Levitt, M. J., Guacci-Franco, N., \& Levitt, J. L. (1993). Convoys of social support in middle childhood and early adolescence: Structure and function. Developmental Psychology, 29, 811-818. 
Lieberman, M., Doyle, A., \& Markiewicz, D. (1999). Developmental patterns in security of attachment to mother and father in late childhood and early adolescence: Associations with peer relations. Child Development, 70(1), 202-213.

Lux, U., \& Walper, S. (2019). A systemic perspective on children's emotional insecurity in relation to father: Links to parenting, interparental conflict and children's social well-being. Attachment \& Human Development, 21(5), 467-484.

Marci, T., Lionetti, F., Moscardino, U., Pastore, M., Calvo, V., \& Altoé, G. (2018). Measuring attachment security via the Security Scale: Latent structure, invariance across mothers and fathers and convergent validity. European Journal of Developmental Psychology, 15(4), 481-492.

Pastore, M. (2016). Overlapping: Estimation of overlapping in empirical distributions. R package version 1.2. Retrieved from http://CRAN.R-project.org/package=overlapping

Pinto, A., Veríssimo, M., Gatinho, A., Santos, A. J., \& Vaughn, B. E. (2015). Direct and indirect relations between parent-Child attachments, peer acceptance, and self-esteem for preschool children. Attachment \& Human Development, 17(6), 586-598.

Posada, G., Lu, T., Trumbell, J., Kaloustian, G., Trudel, M., Plata, S., ... Lay, K. L. (2013). Is the secure base phenomenon evident here, there, and anywhere? A cross-cultural study of child behavior and experts' definitions. Child Development, 84, 1896-1905.

Reid, M., Landesman, S., Treder, R., \& Jaccard, J. (1989). "My family and friends": Six- to twelve-yearold children's perceptions of social support. Child Development, 60, 896-910.

Rosseel, Y. (2012). Lavaan: An R package for structural equation modeling. Journal of Statistical Software, 48(2), 1-36. http://www.jstatsoft.org/v48/i02/

Rothbaum, F., Weisz, J., Pott, M., Miyake, K., \& Morelli, G. (2001). Deeper into attachment and culture. American Psychologist, 56(10), 827-829.

Saarni, C. (1999). The Guilford series on social and emotional development. The development of emotional competence. New York, NY: Guilford Press.

Sarracino, D., Presaghi, F., Degni, S., \& Innamorati, M. (2011). Sex-specific relationships among attachment security, social values, and sensation seeking in early adolescence: Implications for adolescents' externalizing problem behavior. Journal of Adolescence, 34, 541-554.

Seibert, A. C., \& Kerns, K. A. (2009). Attachment figures in middle childhood. International Journal of Behavioral Development, 33(4), 347-355.

Shmueli-Goetz, Y., Target, M., Fonagy, P., \& Datta, A. (2008). The child attachment interview: A psychometric study of reliability and discriminant validity. Developmental Psychology, 44, 939-956.

Shrout, P. E., \& Fleiss, J. L. (1979). Intraclass correlations: Uses in assessing rater reliability. Psychological Bulletin, 86(2), 420-428.

Steele, H., \& Steele, M. (2005). The construct of coherence as an indicator of attachment security in middle childhood: The friends and family interview. In K. A. Kerns \& R. A. Richardson (Eds.), Attachment in middle childhood (pp. 137-160). New York: Guilford Press.

Steele, H., Steele, M., \& Fonagy, P. (1996). Associations among attachment classifications of mothers, fathers, and their infants. Child Development, 67(2), 541-555.

Triandis, H. C. (1995). Individualism and collectivism. Boulder, CO: Westview Press.

van IJzendoorn, M. H., \& Bakermans-Kranenburg, M. J. (2010). Invariance of adult attachment across gender, age, culture, and socioeconomic status? Journal of Social and Personal Relationships, 27 (2), 200-208.

van IJzendoorn, M. H., Moran, G., Belsky, J., Pederson, D., Bakermans-Kranenburg, M. J., \& Kneppers, K. (2000). The similarity of siblings' attachments to their mother. Child Development, 71(4), 1086-1098.

van IJzendoorn, M. H., \& Sagi, A. (1999). Cross-cultural patterns of attachment: Universal and contextual dimensions. In J. Cassidy \& P. R. Shaver (Eds.), Handbook of attachment: Theory, research, and clinical applications (pp. 713-734). New York, NY: The Guilford Press.

Van Ryzin, M., \& Leve, L. (2012). Validity evidence for the Security Scale as a measure of perceived attachment security in adolescence. Journal Adolescence, 35(2), 425-431.

Verschueren, K., \& Marcoen, A. (2002). Perceptions of self and relationship with parents in aggressive and nonaggressive rejected children. Journal of School Psychology, 40(6), 501-522. 
Verschueren, K., \& Marcoen, A. (2005). Perceived security of attachment to mother and father: Developmental differences and relations to self-worth and peer relationships at school. In K. A. Kerns \& R. A. Richardson (Eds.), Attachment in middle childhood (pp. 212-230). New York, NY: Guilford.

Vorria, P., Papaligoura, Z., Dunn, J., van IJzendoorn, M. H., Steele, H., Kontopoulou, A., \& Sarafidou, Y. (2003). Early experiences and attachment relationships of Greek infants raised in residential group care. Journal of Child Psychol Psychiatry, 44(8), 1208-1220.

Waters, E., \& Cummings, E. M. (2000). A secure base from which to explore close relationships. Child Development, 71, 164-172.

Waters, E., \& Deane, K. E. (1985). Defining and assessing individual differences in attachment relationships: Q-methodology and the organization of behavior in infancy and early childhood. Monographs of the Society for Research in Child Development, 50, 41-65.

Waters, E., Hamilton, C. E., \& Weinfield, N. S. (2000). The stability of attachment security from infancy to adolescence and early adulthood: General introduction. Child Development, 71(3), 678-683.

Waters, E., Kondo-lkemura, K., Posada, G., \& Richters, J. E. (1991). Learning to love: Mechanisms and milestones. In M. R. Gunnar \& L. Sroufe (Eds.), Self-processes and development (pp. 217-255). Hillsdale, NJ: Lawrence Erlbaum Associates.

Yu, C. Y. (2002). Evaluating cutoff criteria of model fit indices for latent variable models with binary and continuous outcomes (Unpublished doctoral dissertation). University of California, Los Angeles, CA.

Yu, C. Y., \& Muthen, B. (2002). Evaluation of model fit indices for latent variable models with categorical and continuous outcomes. In Annual meeting of the American Educational Research Association, New Orleans, LA.

Zeanah, C. H., Smyke, A. T., Koga, S. F., \& Carlson, E. (2005). Attachment in institutionalized and community children in Romania. Child Development, 76(5), 1015-1028. 